\title{
Topology and Geometry for Small Sample Sizes: An Application to Research on the Profoundly Gifted
}

\author{
Colleen M. Farrelly \\ Independent Researcher \\ cfarrelly@med.miami.edu
}

\begin{abstract}
This study aims to confirm prior findings on the usefulness of topological data analysis (TDA) in the analysis of small samples, particularly focused on cohorts of profoundly gifted students, as well as explore the use of TDA-based regression methods for statistical modeling with small samples. A subset of the Gross sample is analyzed through supervised and unsupervised methods, including 16 and 17 individuals, respectively. Unsupervised learning confirmed prior results suggesting that evenly gifted and unevenly gifted subpopulations fundamentally differ. Supervised learning focused on predicting graduate school attendance and awards earned during undergraduate studies, and TDA-based logistic regression models were compared with more traditional machine learning models for logistic regression.

Results suggest 1 ) that TDA-based methods are capable of handing small samples and seem more robust to the issues that arise in small samples than other machine learning methods and 2) that early childhood achievement scores and several factors related to childhood education interventions (such as early entry and radical acceleration) play a role in predicting key educational and professional achievements in adulthood. Possible new directions from this work include the use of TDA-based tools in the analysis of rare cohorts thus-far relegated to qualitative analytics or case studies, as well as potential exploration of early educational factors and adult-level achievement in larger populations of the profoundly gifted, particularly within the Study of Exceptional Talent and Talent Identification Program cohorts.
\end{abstract}

\section{Introduction}

There has been an explosion of research related to the educational needs and career achievement of the profoundly gifted in recent years (Farrelly, 2017a; Gross, 2007; Kell, Lubinkski, \& Benbow, 2013; Makel et al., 2016), including both quantitative studies on larger populations (of 200+ participants) and qualitative studies on smaller samples, including the Ruf (Ruf, 2005) and Gross samples (Gross, 2003). Taken as a whole, these studies imply the existence of two disparate types of profound giftedness: 1) the unevenly gifted (who are much more common in the general population and have one or two distinct strengths) and 2) the evenly gifted (whose gifts span the humanities and the sciences/math/technology). This is briefly highlighted in an early study by Lubinski et al. (2001) and reiterated within the Ruf study (Ruf, 2005); a recent analysis on a subset of the Ruf population using advanced methods from a nascent field of machine learning called topological data analysis confirmed this finding quantitatively (Farrelly, 2017a). 
Small sample sizes are the rule in research on rare populations from medicine to psychology (Ayers \& Codell, 2010; Farrelly et al., 2017; Heidema et al., 2006), and research on the profoundly gifted is no different (Gross \& van Vliet, 2005). Samples generally include fewer than 500 participants, with some samples as small as 10 or 20 (Gross, 1992; Gross, 2003). Much of the literature outside the Duke Talent Identification Population (TIP) and Johns Hopkins Study of Exceptional Talent (SET) focuses on small samples using case studies and qualitative methods (Kell, Lubinski, \& Benbow, 2013; Makel et al., 2016), as most statistical methods (and more recent machine learning methods) fail on samples of this size (McCullagh, 1984). The need for quantitative tools to test and understand these small samples is real and urgent, as studies of rare populations are unlikely to yield samples large enough for traditional statistical modeling and even some of its extensions through machine learning (Heidema et al., 2006).

The Farrelly (2017a) paper suggests that clustering methods from a special subfield of machine learning not only handle this small of a sample but seem to excel, finding important insights and offering robustness against noise through the use of multiple thresholds. Applications of this topological approach to logistical models has shown stability and robustness across relatively small sample sizes, as well (Augugliaro, Mineo, \& Wit, 2009; Farrelly, 2017b). It is thought that some of the robustness is rooted in the thresholding effect, called filtration in topological data analysis (TDA) and the general field of topology (Farrelly et al., 2017), and the theoretical results related to relationships between shapes and the functions defined on them, which underlies much of topology in general.

The aims of this study are two-fold. First, this study aims to replicate the Farrelly (2017a) results on a new sample, the original Gross sample along with two of young women added to the follow-up edition of her book. Similar results imply both the stability of the proposed TDA tools on small samples and the distinction between the two types of profound giftedness. The second aim explores the efficacy of several topologically-based logistic regression models on sample outcomes (graduate study and winning top awards at the university or professional level) based on educational interventions and early academic achievement scores documented by Gross (Gross, 2003). This second part not only endeavors to demonstrate the potential of topologically-based logistic regression models on small samples but also seeks to replicate some of the findings related to educational achievement results and the identification of so-called wranglers, individuals whose adult-level achievement surpasses even profoundly gifted peers, found in the most recent follow-up studies of the TIP and SET populations (Kell, Lubinski, \& Benbow, 2013; Makel et al., 2016). The success of topological data analysis tools on small samples and the congruence of their results with larger quantitative studies intimates their usefulness in future studies of the profoundly gifted, as well as in studies of rare diseases, unusual occupational exposures, and other subfields in which small sample are the norm.

\section{Methods \\ Overview of Topological Data Analysis and Models Used General Overview}

TDA is a catch-all term for methods that compute shape-based properties and explore their relationships between datasets (Edelsbrunner \& Harer, 2008; Chen, Genovese, \& Wasserman, 2017). Because these methods are rooted in a field of graduate-level mathematics, an intuitive overview will be presented to give a general introduction to the tools, with references providing more detail. 
One of the central concepts in TDA is homology (Edelsbrunner \& Harer, 2008), which focuses on counting and classifying holes within a given object. For instance, a basketball has a hollow interior, while a baseball does not (figure 1). Topology has a system to track the extant holes for classification (called Betti numbers), which differ for these two balls. Counting holes begins with the number of connected components (no breaks in a surface) and proceeds to higher-dimensional voids (circles, voids, higher-dimensional voids...). For instance, the basketball has a connected component (the skin of the basketball) and a void inside the ball, corresponding to a Betti sequence of $1,0,1,0 \ldots$ (1 connected component, 0 circles, 1 void of 2 dimensions, 0 voids of higher dimensions). The baseball only has a connected component; its Betti number sequence is $1,0 \ldots$ ( 1 connected component and 0 holes whatsoever). These Betti numbers allow topologists to classify objects, as well as study how those objects differ from each other.
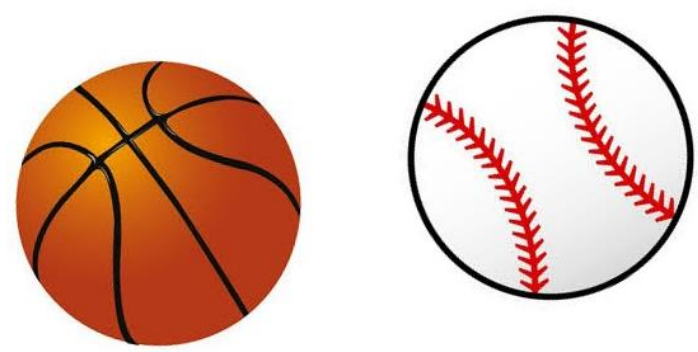

Figure 1: Example of homology

\section{Unsupervised Learning Methods}

Persistent homology extends this concept and can be applied to datasets through several software packages (Edelsbrunner \& Harer, 2008). Persistent homology slices data into different strata, tracking the Betti numbers across slices as if it were an MRI of the data. In this way, sequences of Betti numbers can be tracked through the data MRI, providing insight into the largeness or smallness of a particular hole. Holes across many layers indicate important features, while holes that appear and disappear quickly signal possible noise. Defined strata are created by defining a series of distance thresholds between points to connect them into a series of nested graphs or higher-dimensional objects called simplicial complexes. Simplicial complexes extend typical graphs through connections between 3 or more points (forming triangles, tetrahedra, and higher-dimensional tetrahedra).

By slicing the data, this method can capture higher-dimensional holes, rather than just connected components in a graph (figure 2). Figure 2 defines holes as triangles that are not filled with purple or points not connected by lines. The number of connected components is 3 ( 2 graphs with multiple connected points and 1 lone point). The unfilled triangle in the bottom right corresponds to the next hole number, and there is only one in the simplicial complex. In this example, no higher-dimensional holes are present, yielding this sequence of Betti numbers: $3,1,0 \ldots$ 


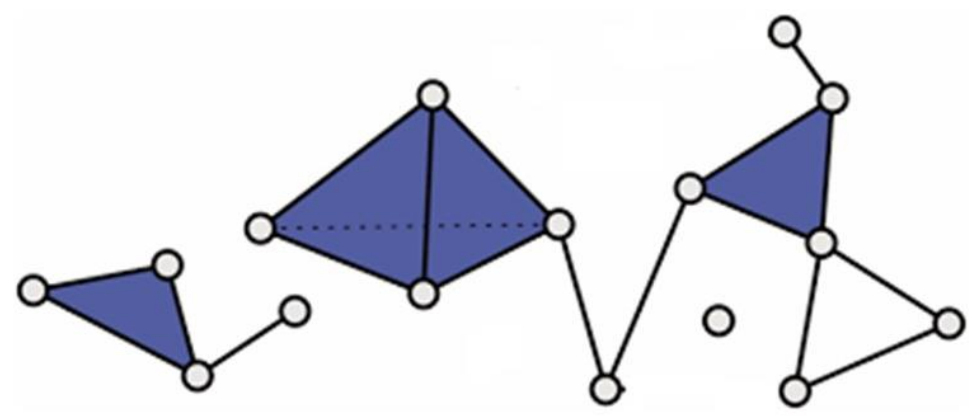

Figure 2: Example of simplicial complex built from data

These sequences of Betti numbers are then plotted to show changes across slices, typically through a barcode or persistence diagram. Persistence diagrams allow for a single plot to capture persistence of all topological features identified across slices and dimensions. However, high-dimensional features are rare and typically don't persistent across many slices (and, thus, are noise in the data); the first two Betti numbers are usually sufficient to capture most relevant features of the data.

The sequence of connected components across the data slices has a curious relationship to another machine learning method called single-linkage hierarchical clustering, in which clusters at each height level correspond to the connected components at that slice. When both techniques employ the same distance metric, results are identical between the two techniques (Farrelly et al., 2017; Lee et al., 2012). These two options come with their own plots and statistical tests, and, thus, a researcher might favor one over the other for a given project. One method might have a more familiar explanation or visualization for a given audience (persistence diagrams or barcodes vs. dendrograms or heat maps). Statistical tests exist to compare results of either method, allowing for validation of samples and comparison of important structures in the data. A null distribution or permutation distribution provides a comparison for a given diagram or dendrogram, following by the computation of Wasserstein distances between persistence diagrams or Hausdorff distance between dendrograms to derive a $p$ value from the null distribution (Farrelly et al., 2017).

This study compares persistence diagrams and hierarchical clustering dendrograms between the current and previous Farrelly (2017a) sample using the TDA package in R (for persistence diagrams) and the hclust function in $\mathrm{R}$ (for dendrograms), where nonparametric tests were written from scratch in $\mathrm{R}$ following Farrelly et al., 2017.

An alternative to this type of clustering involves a TDA technique called Morse-Smale clustering (Chen, Genovese, \& Wasserman, 2017; Gerber et al., 2013). Loosely, Morse-Smale clustering filters data with a function (called a Morse function) and decomposes the data space based on the Morse function's peaks and valleys (Gerber et al., 2013). In this way, the filtering function is analogous to slicing a data landscape based on height functions (figure 3). As the function changes value, it creates flow from peaks (areas where height value is large) to valleys (areas where height value is small), which connects peaks to valleys. Areas that share connections are limned, and these areas form landscape partitions. In figure 3 , the space likely forms a single partition, as only one peak exists.

A discrete version of this exists for use on data, where data points are clustered based on shared function peaks and valleys (Gerber et al., 2013). The msr R package implements a version of this 
algorithm and is used in this paper to obtain Morse-Smale clusters. One advantage of Morse-Smale clustering is the ability to visualize group differences on a set of variables, allowing for easy visualization of which predictors seem to separate well in the clustering.

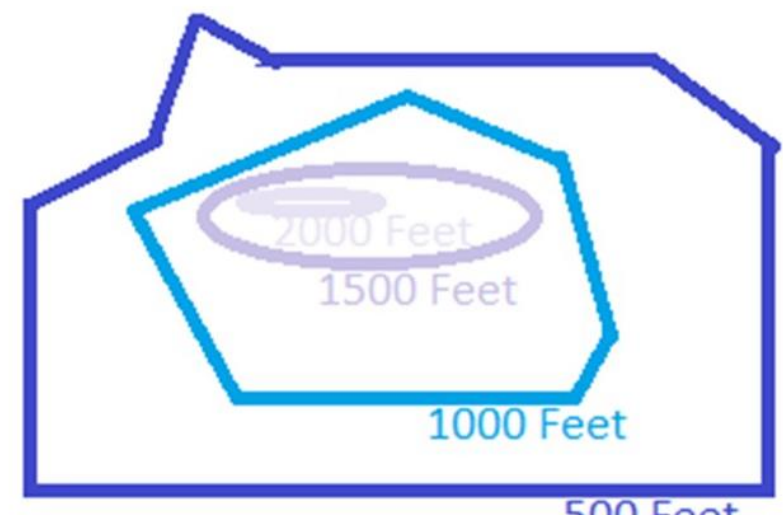

\section{Feet}

Figure 3: Example of a filter function based on landscape height

\section{Supervised Learning Methods}

\section{a) Logistic Regression}

Logistic regression is an extension of multivariate regression to binary outcomes (yes/no, healthy/disease...) through the use of a link function (McCullagh, 1984). A common choice of link in the social sciences is the logit link, where the coefficients of the fitted logistic regression model can be used to find the odds ratio for a particular term (McCullagh, 1984). This model falls under the framework of generalized linear models, inheriting the limitations of this framework, including sample size requirements (Heidema et al., 2006; McCullagh, 1984). In many datasets studying rare populations, sample size may pose issues to this method, and extensions of this general framework are needed (Farrelly, 2017b; Heidema et al., 2006).

This study compares two types of logistic regression extensions to explore efficacy on small datasets: 1 ) machine learning algorithms (multivariate adaptive regression splines and Bayesian model averaging ensembles) and 2) topologically-based extensions of penalized logistic regression models (homotopy LASSO and DGLARS).

\section{b) Penalized Regression Models}

Penalized linear models, such as the LASSO model, extend logistic regression to samples in which predictors may outnumber observations ( $p>n$ condition) by adding a penalty term to the original least squares estimator that shrinks small coefficients to 0 (Wu et al., 2009; Zou \& Hastie, 2005). Geometrically, it is a bit like a cowboy standing at the origin casting his lasso rope to capture any predictors within range of the rope, removing them from the set.

\section{c) Homotopy LASSO}

The calculations driving LASSO can get stuck in local peaks and valleys in the data, resulting in inaccurate estimated coefficients (Osborne, Presnell, \& Turlach, 2000). Homotopy LASSO (HLASSO) provides a fix to 
this problem, employing a topologically-based search for model coefficient estimation to begin with a known solution to a similar problem (that doesn't have these local obstacles) and slowly deform that solution to a target equation that includes the less-nice problem within the data (broadly called a homotopy method). Thus, homotopy search avoids geometric traps in the data space (Osborne, Presnell, \& Turlach, 2000). Analogously, these algorithms are a bit like providing a guide rope to a blindfolded person tasked with finding a target somewhere in a field of obstacles. Homotopy methods tether the starting point and the final target.

\section{d) DGLARS}

Much like LASSO models, least angle regression (LARS) models utilize a penalty term to build generalized linear regression models (Augugliaro, Mineo, \& Wit, 2009). Coefficients are initially set to 0 , and correlated predictors are added stage-wise, starting with the highest correlation term. These estimates are then increased in the direction of their correlation until the algorithm uncovers another predictor with a greater outcome correlation. When multiple terms start entering the model, these terms' estimates are jointly increased until all predictors have entered the model; then, the optimal solution is chosen among the models according to best fit criteria (AIC or BIC, for instance). Thus, LARS is similar to forward regression selection schemes only within a penalized framework.

A recent extension of LARS, DGLARS, leverages the data space's geometry (called the error tangent space) to scale the generalized linear model at each update according to a metric termed the conditional Fisher information, giving a conditional Rao's score statistic (Augugliaro, Mineo, \& Wit, 2009). When the log-likelihood function gives a flat geometry, this score statistic will equal 0 ; it will be dropped from the solution equation, forming a set of non-selected model coefficients. Non-zero score statistics are then ranked by absolute value in the separate set of selected model coefficients. Some conditions are imposed on the model and relationships between model coefficients to ensure that collinear predictors are not added (called the equiangularity condition), and the penalty term allows the model to deal with small sample sizes.

Essentially, the loglikelihood space's inherent geometry partitions predictors into 3 sets: selected predictors, redundant predictors (identified through the equiangularity condition), and non-selected predictors. This is analogous to a geologist sorting rock samples into different bags (igneous, metamorphic, and sedimentary) based on the visible properties of a given rock. He can then examine the rocks in the correct bag to see which matches his lab sample.

\section{e) Multivariate adaptive regression splines}

Multivariate adaptive regression splines (MARS) models build generalized linear models, such as logistic regression, by creating a weighted sum of special functions, called basis functions (Friedman, 1991). Basis functions wiggle lines between points, similar to a downhill skier's carving a path between target flags (Friedman, 1991). Thus, MARS builds a flexible model in which predictors can have nonlinear relations with an outcome and deal with small sample sizes by ignoring some predictors when choosing the best basis functions. This is similar to a slalom skier hitting each flag on his way down but having the flexibility to choose his best route between flags, allowing him to minimize his final time.

\section{f) Bayesian Model Averaging}


Another logistic regression extension is Bayesian model averaging (BMA), where all possible logistic regression models created from a set of predictors are combined into a weighted average according to its Bayes factor (B), which compares that particular model to the null model as a way to rank information gain (Raftery, Madigan, \& Hoeting, 1997). This method has several advantages, which include its ensemble formulation (created similarly to boosting ensembles) and its ranking of solution models, allowing the user to can determine if one model is clearly best given a criterion (i.e. BIC, AIC...) or if several models seem to fit the data equally well (Raftery, Madigan, \& Hoeting, 1997). It is a bit like a painter blending palette colors to derive the right hue for a painting; some hues may be more complicated to create than others from a given palette. Because the models within the ensemble are logistic regression models, BMA suffers from the same limitations as single logistic regression models, including sample size limitations.

\section{Dataset}

The sample used in the unsupervised and supervised analyses include the original Gross sample of 15 individuals selected across Australian states with Stanford-Binet LM measured IQs ranging from 162 to 200+, including three individuals whose IQs hit the ceiling of the test (Gross, 2003). These participants all had documented achievement scores in math and reading recorded at the time of the study. Per Gross's records, achievement scores at the time of the study are measured in years ahead of age, with a top cutoff of 5 , such that any child 5 or more years ahead in reading or math was coded as 5 years ahead.

All but one participant (Jade) had additional educational outcomes tracked through college and graduate school (alterations to educational path, entering graduate programs, and winning major awards during college), and all measured achievement scores at the time of the original study. In addition to the original Gross sample, two younger women (Sally and Gena) were added whose educational trajectories were well-documented and whose Stanford-Binet LM scores had been measured during childhood (Gross, 2003). Achievement scores were estimated at $5+$ years ahead based on the reported SAT scores they obtained in early adolescence.

All 17 participants were included for unsupervised learning tasks, and the 16 participants with educational outcome data were included in the supervised learning tasks aimed at predicting graduate school attendance and receiving major academic awards during their undergraduate studies. Similar to the SET and TIP populations, 9 of the 16 participants enrolled in graduate programs (56\%), with 5 of the 16 already in or finished with PhD programs (31\%) at the time of follow-up. 2 additional participants opted out of further education to pursue career opportunities. 4 participants received at least one major award by the time of follow-up, including university medals in their fields and awards to study at top graduate programs abroad (such as Fulbright Scholarships).

\section{Analyses}

To explore ability tilt and attempt to replicate the findings in the Farrelly (2017a) study, the full sample was analyzed through persistent homology and Morse-Smale clustering using Stanford-Binet LM scores, childhood math achievement scores, and childhood reading achievement scores. Persistent homology 
was implemented through the TDA R package using the ripsDiag() function with filtrations set to 10 and computation of the $0^{\text {th }}, 1^{\text {st }}$, and $2^{\text {nd }}$ Betti numbers computed; the persistence diagram was then plotted. The $0^{\text {th }}$ Betti numbers were also computed through the dendrogram produced with single-linkage hierarchical clustering methods through the hclust() function from the Base R package. Morse-Smale clustering was done through the $\mathrm{msr}($ ) package, utilizing the msc.nn() function with $\mathrm{k}=5$ and $\mathrm{k}=10$; pLevel was set to 0.5 . Standard plots were obtained with through the $\mathrm{msr}($ ) package, and a dimensionality reduction plot called multidimensional scaling ( $R$ base package) was used produce a 2-dimensional plot of the Morse-Smale clusters to examine the quality of group separation.

To explore the efficacy of TDA-based methods in supervised learning on small samples, 2 logistic regression models were created to model graduate school attendance and awards achieved during undergraduate studies for the 16 participants with outcome data. Predictors included Stanford-Binet LM scores, childhood math achievement scores, childhood reading achievement scores, and educational trajectory data (early entrance indicator, grade-skip indictor, subject acceleration indictor, and radical acceleration indicator). Machine learning models run included a MARS logistic regression model with default parameters ( $R$ package earth), BMA logistic regression model with default parameters ( $R$ package BMA), DGLARS logistic regression model with default parameters ( $R$ package dglars), and homotopy-based LASSO with default parameters ( $R$ package lasso2). Model fit statistics, confidence intervals, and model fit plots were obtained for each package including these options for a given model to understand each method's potential limitations or strengths on small datasets.

\section{Results}

\section{Unsupervised Learning Results}

Similar to previous results, an early split between evenly and unevenly gifted participants emerges in the $0^{\text {th }}$ Betti number, corresponding to distinct differences between the two groups and replicating previous results (figure 4). The dendrogram shows that this split includes the 4 participants with a measured Stanford-Binet LM IQ greater than 200-Adrian, lan, Christopher, and Gena, all of whom were also 5+ years ahead in both reading and math skills (figure 5 ). The less-separated branch of unevenly gifted participates includes a partition with 3 students who scored very high on the Stanford-Binet LM (170+) but, at the time of the first study, largely hadn't had access to acceleration and educational accommodations, logging lower achievement scores than those with similar IQ scores who had more access to educational alternatives. 
Intelligence and Achievement Persistence Diagram

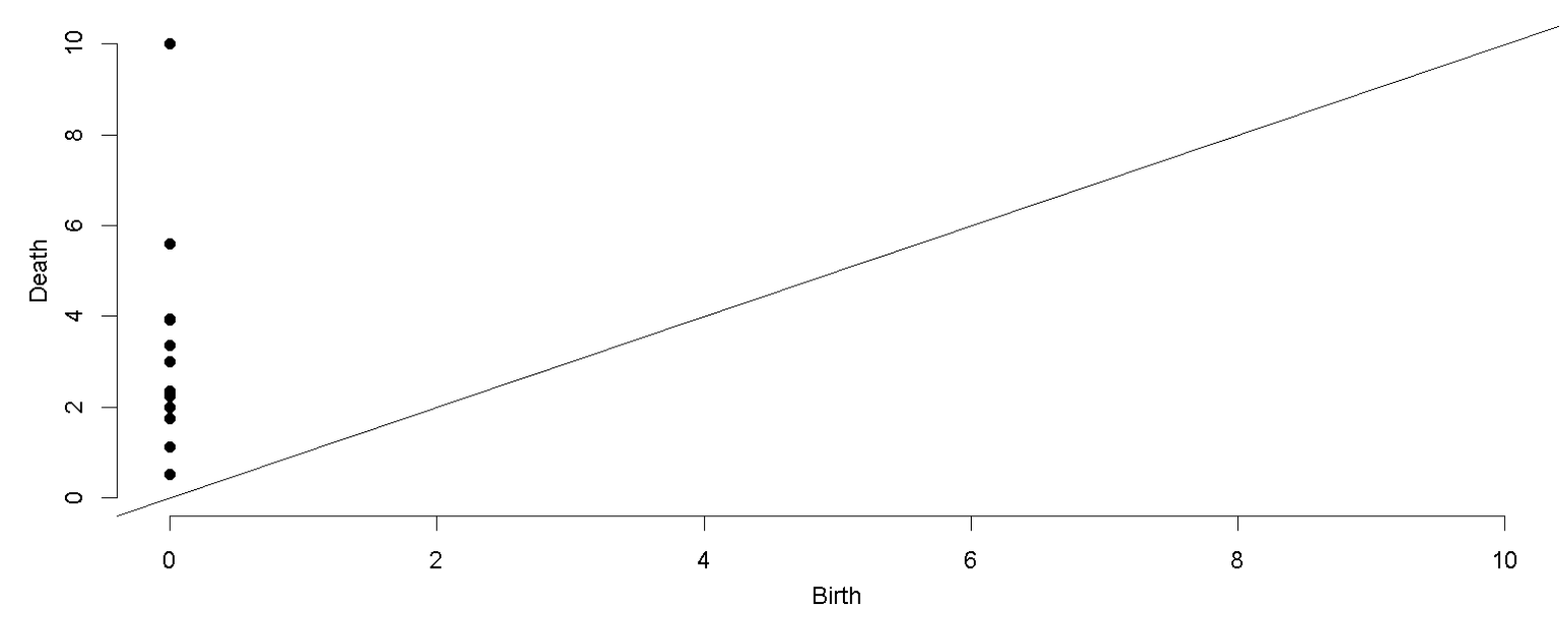

Figure 4: Persistence Diagram of Gross sample

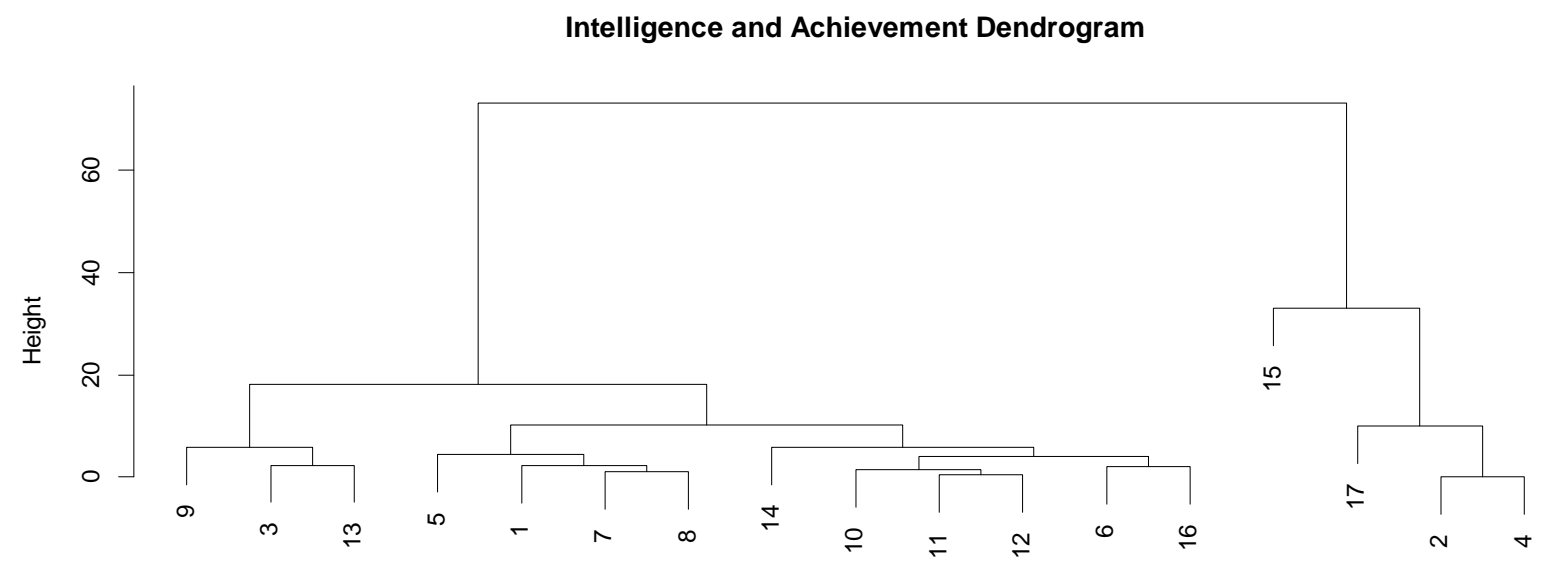

Figure 5: Dendrogram of Gross sample

The separation among the unevenly gifted is a bit less-defined than in the previous sample, and this may be related to the current study's much smaller sample size (17 vs. 31). However, nonparametric testing of differences in the persistence diagrams and dendrograms between the 2 cohorts (set-up as in the Farrelly et al., 2017, paper) revealed no significant differences. In addition, some participants' StanfordBinet LM IQ scores are suspect, as testers stopped before the participant hit a ceiling on the test. However, the general trend seems to hold with previous results, bolstering the evidence for distinct subpopulations based on relative strengths within the profoundly gifted population.

The Morse-Smale cluster results support the fracturing seen in the persistent homology and hierarchical clustering results, with several distinct subgroups identified for both the $k=5$ and $k=10$ trials. In the $k=5$ trial (including $~ 33 \%$ of the sample as nearest neighbors), 3 of the 4 individuals with an IQ>200 and advancement of $5+$ years in each area separate out into their own subgroups, while the other groups coalesce according to relative strengths and advancement (figure 6 , table 1). At $k=10$ (including $>50 \%$ of the sample as nearest neighbors), the results are muddied, with groups largely separating based on 
Stanford-Binet LM IQ (table 2). The low-dimensional plot shows a wide variation of component scores within groups, though identified groups do seem to be sufficiently separated by the first two MDS components.

Morse-Smale Cluster Result MDS Plot Intelligence and Achievement

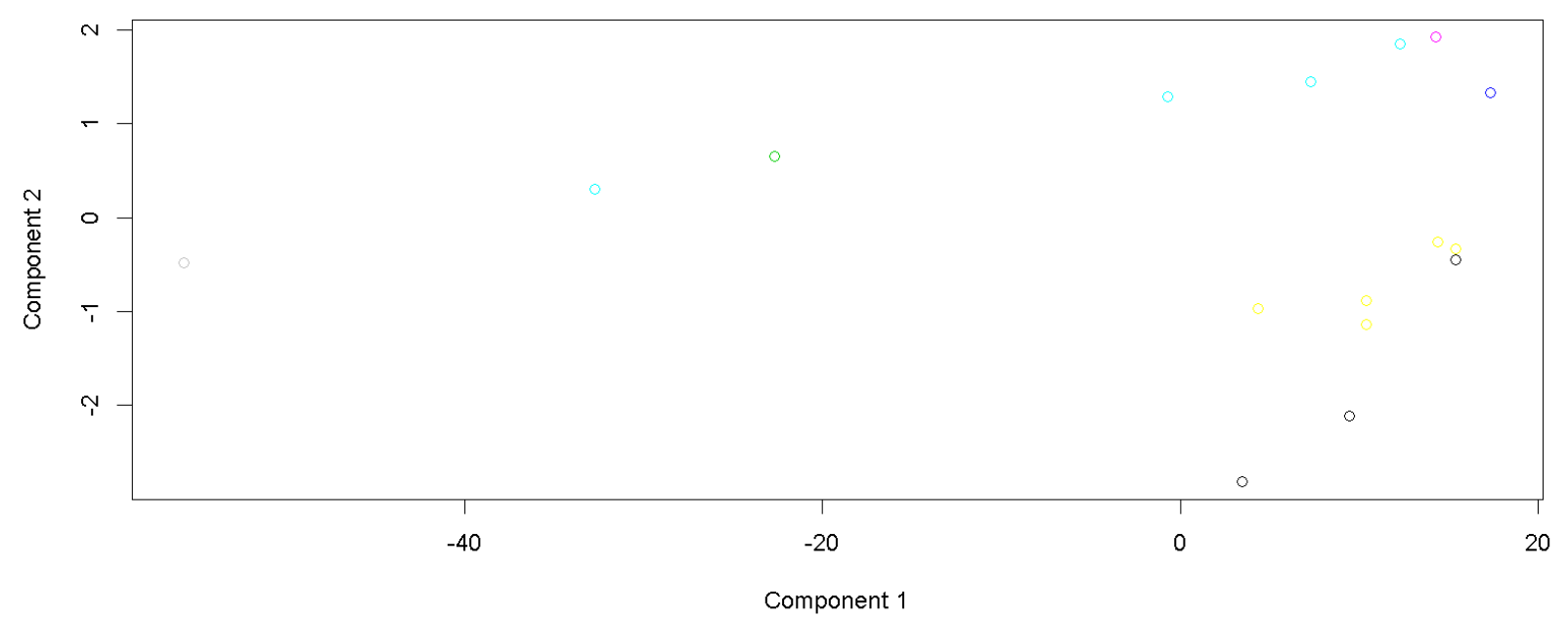

Figure 6: Morse-Smale clustering results with $k=5$

Table 1: Morse-Smale clustering results summary $k=5$

\begin{tabular}{|l|l|l|l|l|}
\hline Row Labels & $\begin{array}{l}\text { Average of } \\
\text { SBLM }\end{array}$ & $\begin{array}{l}\text { Average of } \\
\text { English }\end{array}$ & $\begin{array}{l}\text { Average of } \\
\text { Math }\end{array}$ & $\begin{array}{l}\text { Number in } \\
\text { Group }\end{array}$ \\
\hline 1 & 168 & 1.833333333 & 3 & 3 \\
\hline 2 & 200 & 5 & 5 & 1 \\
\hline 3 & 200 & 5 & 5 & 1 \\
\hline 4 & 160 & 5 & 2 & 1 \\
\hline 5 & 180.75 & 5 & 4.625 & 4 \\
\hline 6 & 163 & 5 & 5 & 1 \\
\hline 7 & 166.4 & 2.6 & 4.2 & 5 \\
\hline 8 & 233 & 5 & 5 & 1 \\
\hline
\end{tabular}

Table 2: Morse-Smale clustering results summary $k=10$

\begin{tabular}{|l|l|l|l|l|}
\hline Row Labels & $\begin{array}{l}\text { Number } \\
\text { in Group }\end{array}$ & $\begin{array}{l}\text { Average of } \\
\text { SBLM }\end{array}$ & $\begin{array}{l}\text { Average of } \\
\text { Math }\end{array}$ & $\begin{array}{l}\text { Average of } \\
\text { English }\end{array}$ \\
\hline 1 & 2 & 171 & 3 & 1.25 \\
\hline 2 & 5 & 173.8 & 4.3 & 3 \\
\hline 3 & 1 & 200 & 5 & 5 \\
\hline 4 & 9 & 178.2222222 & 4.222222222 & 4.555555556 \\
\hline
\end{tabular}

\section{Supervised Learning Results}


All 4 logistic regression models run on the graduate school outcome logged decent fit statistics, with the MARS model accounting for $86 \%$ of the variance, BMA models ranging from -35 to -39 BIC scores, and DGLARS logging a BIC of 17. MARS plots suggest most individuals fit the model well (figure 7), and the DGLARS plots imply that the model did run into any singularity issues in its computation (figure 8). The BMA results suggest insufficient sample size, as demonstrated by the extremely large confidence intervals obtained by the final ensemble (figure 9).
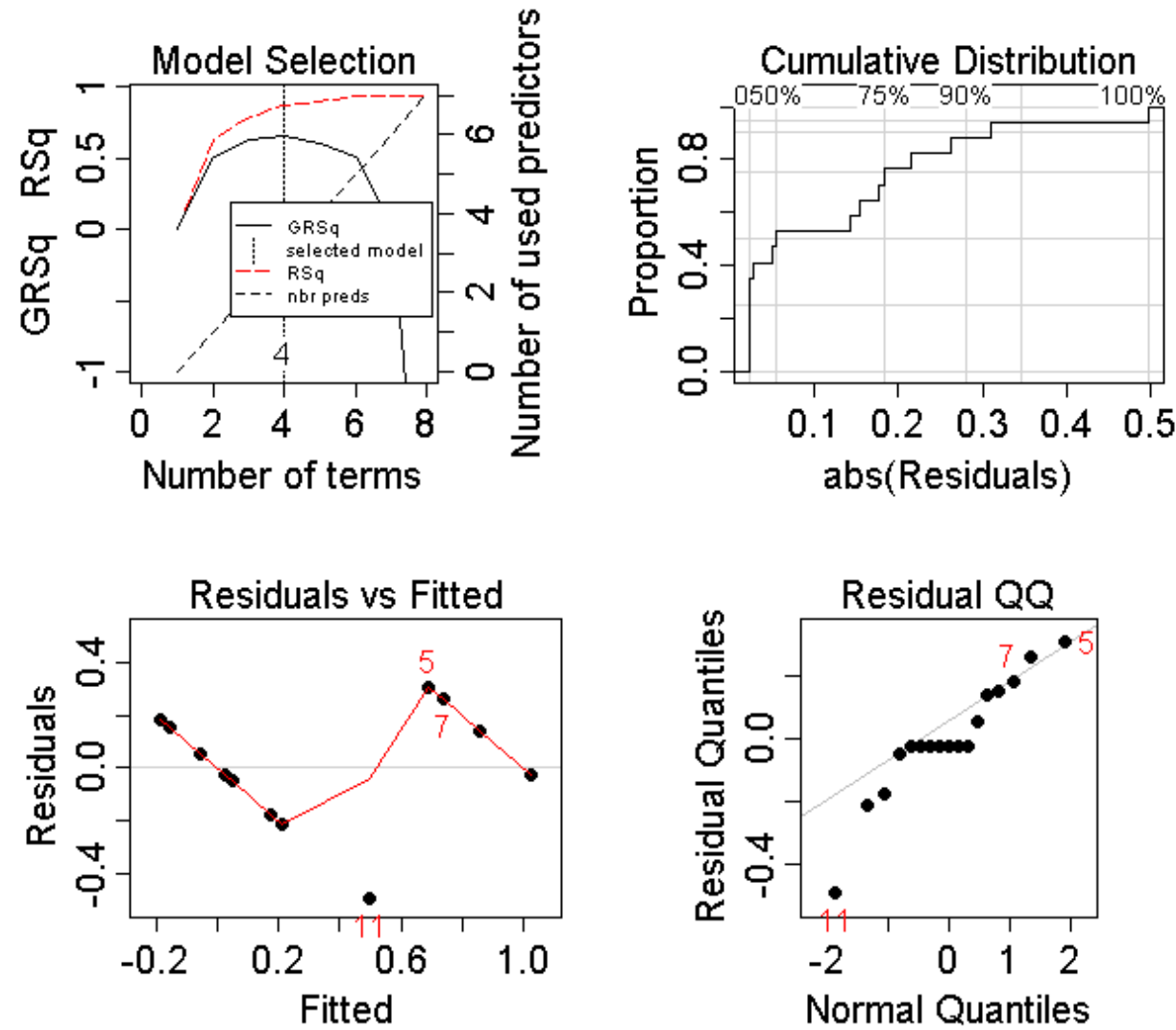

Figure 7: MARS model diagnostic plots
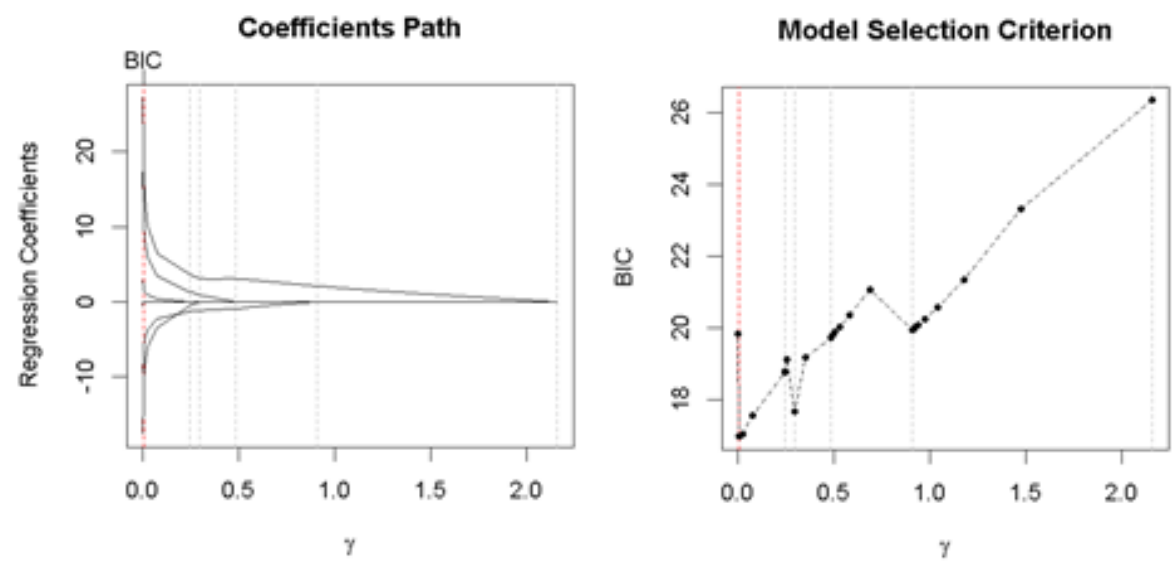

Figure 8: DGLARS coefficient path (diagnostic) plot 

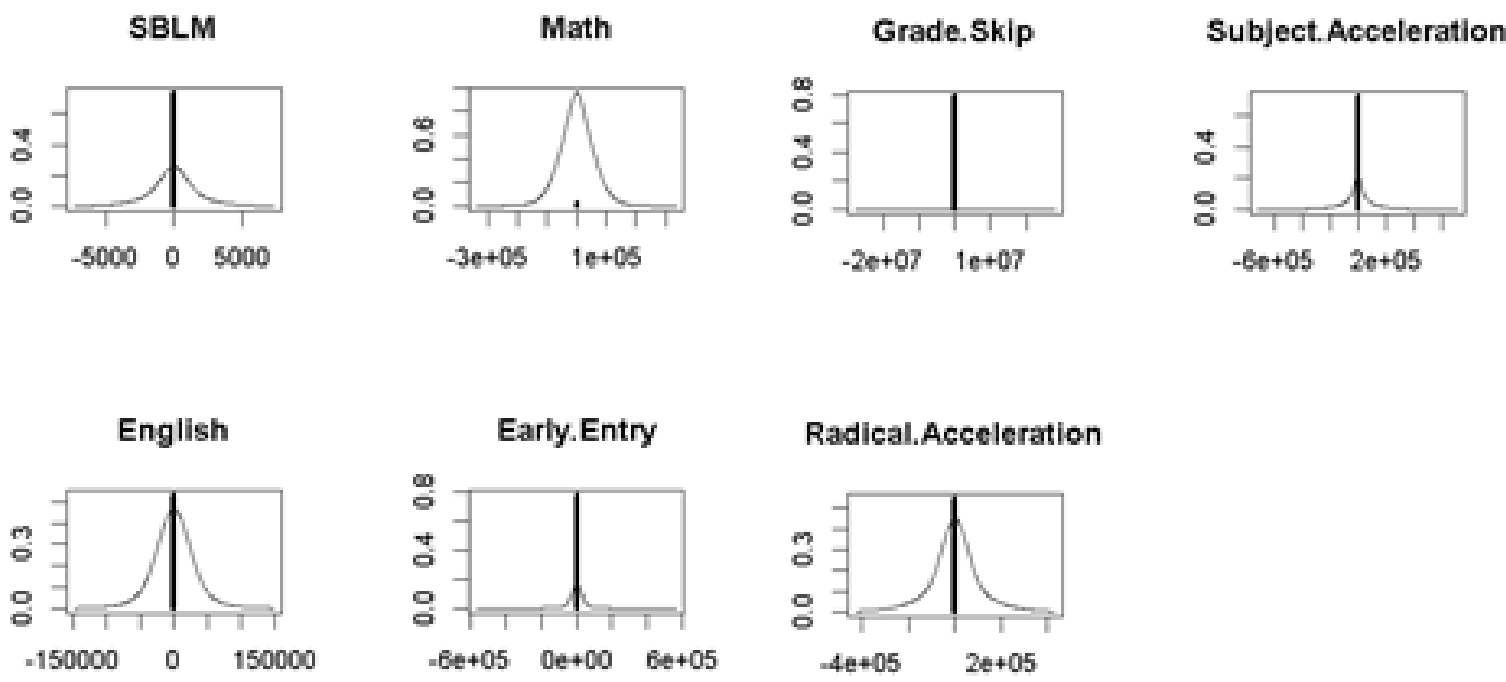

Figure 9: BMA coefficient confidence interval plot

In addition, the BMA and DGLARS models suffer from coefficient inflation (table 3 ), logging coefficients of $>15$ on several factors. It is unlikely that each year of advancement in mathematics gives an odds ratio $>10000$ or that radical acceleration yields an odds ratio of $>20000$ with respect to graduate school attendance. Odds ratios in the MARS and HLASSO models yield more believable results, ranging from 0.5 to 10. The correspondence of selected factors of early advancement in English and math, along with early educational factors such as early entrance and grade skips, suggests that these factors are related to future graduate school attendance and may be of interest in future studies with larger sample sizes.

Table 3: Graduate education model coefficients

\begin{tabular}{|l|l|l|l|l|l|l|l|}
\hline & SBLM & English & Math & $\begin{array}{l}\text { Early } \\
\text { Entry }\end{array}$ & $\begin{array}{l}\text { Grade } \\
\text { Skip }\end{array}$ & $\begin{array}{l}\text { Subject } \\
\text { Acceleration }\end{array}$ & $\begin{array}{l}\text { Radical } \\
\text { Acceleration }\end{array}$ \\
\hline MARS & & 0.26 & 0.33 & -0.49 & & & \\
\hline BMA & 0.54 & 8.68 & 51.71 & -10.07 & -7.55 & 5.70 & 18.95 \\
\hline DGLARS & & 2.00 & 12.76 & -7.17 & -12.92 & & 20.28 \\
\hline HLASSO & 0.05 & 1.13 & 2.38 & -0.68 & -0.76 & & \\
\hline
\end{tabular}

Results of the awards received logistic regression models suggest that the traditional machine learning algorithms, MARS and BMA, struggle, while the topologically-based algorithms, DGLARS and HLASSO, excel on this problem (table 4). The BMA model again suffers from coefficient blow-up and confidence intervals of $>50000$. The MARS model only captures $27 \%$ of the variance and selects only radical acceleration as a predictor associated with awards won later on. DGLARS shows similar BIC estimate (18) and no large odds ratios within this model (early entry $O R=9.03$; radical acceleration $O R=105.6$ ). Both of these numbers correspond well with the estimates from the HLASSO model (ORs of 4.22 and 26.31, respectively). HLASSO also selected Stanford-Binet $L M$ score $(\mathrm{OR}=1.02)$ and English achievement metrics $(\mathrm{OR}=0.77)$ early on as predictors of future accomplishment in undergraduate studies. Given the overlap of early entry and radical acceleration across models, future studies of adult accomplishments may want to incorporate these two factors. 
Table 4: Awards model coefficients

\begin{tabular}{|l|l|l|l|l|l|l|l|}
\hline & SBLM & English & Math & $\begin{array}{l}\text { Early } \\
\text { Entry }\end{array}$ & $\begin{array}{l}\text { Grade } \\
\text { Skip }\end{array}$ & $\begin{array}{l}\text { Subject } \\
\text { Acceleration }\end{array}$ & $\begin{array}{l}\text { Radical } \\
\text { Acceleration }\end{array}$ \\
\hline MARS & & & & & & & 0.44 \\
\hline BMA & & -6.25 & 5.79 & 0.97 & 1.38 & 2.41 & 33.10 \\
\hline DGLARS & & & & 2.20 & & & 4.66 \\
\hline HLASSO & 0.02 & -0.26 & & 1.44 & & & 3.27 \\
\hline
\end{tabular}

\section{Discussion}

This study bolsters support for the use of topological data analysis tools on small datasets, which previously have been relegated to case studies and qualitative analyses in the field of gifted education. Methods such as persistent homology and HLASSO seem to work well on samples as small as 16 or 17 participants, where other methods fail badly.

Also of note, this study found consistent results related to future educational attainment and field of study accomplishments, particularly related to early educational and achievement factors for graduate school attendance and related to radical acceleration and early entrance for later accomplishments in one's chosen field. Because these outcomes have been explored in cohorts with early milestone and educational data available (particularly the SET sample), replication on a larger cohort in which more machine learning models can be run is possible, and confirmation of these results would have substantial implications on educational policy for profoundly gifted students. It seems that early educational intervention and opportunities to advance are crucial for future career achievement and attaining low base-rate accomplishments in a given field. The author suspects these factors may also play a role in the development of so-called wranglers, those who become eminent. One child in this cohort is known to have achieved eminence in his field (Adrian), and his education had substantial alterations starting early on.

One of the limitations of this study, particularly in the supervised learning portion, is the small sample size. What we see here is likely near the limit of even topologically-based methods. However, the replication previous TDA results in a new cohort suggest that these methods can deal with much smaller samples than previous estimated when the underlying structures are well-defined phenomena.

In all, this study confirms the efficacy of TDA methods in studying rare populations in quantitatively rigorous ways, which opens the door for use of these methods in studies of rare disease (including Mendalian disorders), intellectual outliers (both on the high and low ends of the spectrum), unusual exposure cohorts (such as victims of nuclear disasters), and other important problems facing scientists today.

\section{References}

Augugliaro L, Mineo AM, Wit EC. A differential geometric approach to identify important variables in GLMs when $p>>n$. Statistics and the Life Sciences: High-dimensional inference and complex data, University of Groningen, Groningen, September. 2009:9-11. 
Ayers KL, Cordell HJ. SNP Selection in genome-wide and candidate gene studies via penalized logistic regression. Genetic epidemiology. 2010 Dec 1;34(8):879-91.

Chen, Y. C., Genovese, C. R., \& Wasserman, L. (2017). Statistical inference using the Morse-Smale complex. Electronic Journal of Statistics, 11(1), 1390-1433.

Edelsbrunner, H., \& Harer, J. (2008). Persistent homology-a survey. Contemporary mathematics, 453, 257-282.

Farrelly, C. M. (2017a). Topological Data Analysis for Data Mining Small Educational Samples with Application to Studies of the Gifted.

Farrelly, C. M. (2017b). Topology and Geometry in Machine Learning for Logistic Regression.

Farrelly, C. M., Schwartz, S. J., Amodeo, A. L., Feaster, D. J., Steinley, D. L., Meca, A., \& Picariello, S. (2017). The analysis of bridging constructs with hierarchical clustering methods: An application to identity. Journal of Research in Personality, 70, 93-106.

Friedman JH. Multivariate adaptive regression splines. The annals of statistics. 1991 Mar 1:1-67.

Gerber, S., Rübel, O., Bremer, P. T., Pascucci, V., \& Whitaker, R. T. (2013). Morse-smale regression. Journal of Computational and Graphical Statistics, 22(1), 193-214.

Gross, M. U. (1992). The use of radical acceleration in cases of extreme intellectual precocity. Gifted child quarterly, 36(2), 91-99.

Gross, M. U. (2003). Exceptionally gifted children. Routledge.

Gross, M. U. (2006). Exceptionally gifted children: Long-term outcomes of academic acceleration and nonacceleration. Journal for the Education of the Gifted, 29(4), 404-429.

Gross, M. U., \& van Vliet, H. E. (2005). Radical acceleration and early entry to college: A review of the research. Gifted Child Quarterly, 49(2), 154-171.

Heidema AG, Boer JM, Nagelkerke N, Mariman EC, Feskens EJ. The challenge for genetic epidemiologists: how to analyze large numbers of SNPs in relation to complex diseases. BMC genetics. 2006 Apr 21;7(1):23.

Kell, H. J., Lubinski, D., \& Benbow, C. P. (2013). Who rises to the top? Early indicators. Psychological Science, 24(5), 648-659.

Lee, H., Kang, H., Chung, M. K., Kim, B. N., \& Lee, D. S. (2012). Persistent brain network homology from the perspective of dendrogram. IEEE transactions on medical imaging, 31(12), 2267-2277.

Lubinski, D., Webb, R. M., Morelock, M. J., \& Benbow, C. P. (2001). Top 1 in 10,000: a 10-year follow-up of the profoundly gifted. Journal of applied Psychology, 86(4), 718. 
Makel, M. C., Kell, H. J., Lubinski, D., Putallaz, M., \& Benbow, C. P. (2016). When lightning strikes twice: Profoundly gifted, profoundly accomplished. Psychological Science, 27(7), 1004-1018.

McCullagh P. Generalized linear models. European Journal of Operational Research. 1984 Jun 1;16(3):285-92.

Osborne, M. R., Presnell, B., \& Turlach, B. A. (2000). A new approach to variable selection in least squares problems. IMA journal of numerical analysis, 20(3), 389-403.

Raftery AE, Madigan D, Hoeting JA. Bayesian model averaging for linear regression models. Journal of the American Statistical Association. 1997 Mar 1;92(437):179-91.

Ruf, D. L. (2005). Losing our minds: Gifted children left behind. Great Potential Press, Inc..

Wu TT, Chen YF, Hastie T, Sobel E, Lange K. Genome-wide association analysis by lasso penalized logistic regression. Bioinformatics. 2009 Mar 15;25(6):714-21.

Zou H, Hastie T. Regularization and variable selection via the elastic net. Journal of the Royal Statistical Society: Series B (Statistical Methodology). 2005 Apr 1;67(2):301-20. 